\title{
Cloud Computing Applications in Universities
}

\author{
Lei Yan ${ }^{\mathrm{a}, *}$, Yan Wang ${ }^{\mathrm{b}}$, Jiaqi Hao \\ Department of Computing, North China Electric Power University, No. 619 Yonghua North Street, \\ Baoding, China \\ ayanleics@ncepu.edu.cn, bwangyan1206@126.com, ‘huruzhen1566@163.com \\ *corresponding author
}

Keywords: Information Technology, Cloud Computing, University

Abstract: Information technology plays an important role in education, and our country pays more and more attention to the application of information technology. Cloud computing is a technology that has emerged and spread rapidly in recent years. It is an on-demand computing service based on the Internet. The main goal of this paper is to highlight the significance of cloud computing in universities. This paper introduces the paradigms, concepts, levels, types and characteristics of cloud computing. Cloud computing technology has improved the performance of universities and played an important role in reducing costs. In addition, this paper presents the factors that influence universities' adoption of cloud computing.

\section{Definition of cloud computing}

"Cloud computing" is a distributed computing technology based on the Internet ${ }^{[1]}$, which provides storage, software, computing platform, computing infrastructure and other services to target users in a reusable manner on demand, and implements services according to users' consumption conditions. Cloud computing makes computing services simpler and infrastructure less complex.

\subsection{Cloud Computing Layers and Types}

In terms of service delivery, cloud computing is divided into three main layers: Software as a Service (SaaS), Platform as a Service (PaaS), and Infrastructure as a Service (IaaS) ${ }^{[2]}$. There are four types of cloud computing deployments: private cloud, public cloud, community cloud, and hybrid cloud.

\subsubsection{Cloud Computing Layer}

"Software as a product" is the traditional way of distributing software. The SaaS delivery model transforms the concept of "software as a product" into "software as a service". The software or application runs on the cloud (in the provider's server) and the user can access the application over the Internet to work properly ${ }^{[3]}$. Consumers don't need to know the infrastructure to run this application, just work and pay. 
PaaS provides an efficient environment and powerful tools for developers to create and deploy applications. Instead of worrying about infrastructure issues, developers or companies focus on innovation.

IaaS is the base layer for computing resources. It provides on-demand and scalable complete infrastructure resources (such as servers, software, network equipment and storage). IaaS allows users to manage and configure cloud servers just like normal physical servers. With IaaS, consumers will be freed from purchasing the latest technology, maintenance, software upgrades and software licenses.

\subsubsection{Types of Cloud Computing}

In terms of deployment methods, there are four types of cloud computing, namely private cloud, public cloud, community cloud and hybrid cloud. A type of private cloud that runs cloud infrastructure only for a specific organization. A public cloud type is one where cloud infrastructure is available to multiple individuals or organizations. A community cloud type is one where cloud infrastructure is shared by several organizations that share a common focus on a specific issue, such as mission, policy, or security. Cloud infrastructure is managed by these organizations or by third parties. A hybrid cloud is a mix of two or more of the previous types. Organizations can use this type when their infrastructure is under-capacity and needs to expand their infrastructure to meet their business.

\subsection{Features of Cloud Computing}

\subsubsection{Virtualization}

Computing resource virtualization provides a new method to obtain and utilize computing resources remotely ${ }^{[4]}$. No matter where computing resources are, end users can call these resources from terminal devices anytime and anywhere, but do not need to know the location of these resources. Therefore, users can accomplish a lot using cloud computing services.

\subsubsection{High reliability}

Computing service resources provided by cloud providers provide users with stronger reliability than relying on local resources. Cloud providers distribute resource services around the world, not in the same location. Therefore, in any error situation in any cloud location, the cloud service provider can redirect the end user request to the same cloud service located in a different physical location.

\subsubsection{Versatility}

Cloud services are not limited to certain applications. It can serve several parts of different disciplines that exist in the same cloud environment, such as processing pipeline systems, it can convert thousands of word documents to PDF and millions of OCR documents to searchable text form. Therefore, a large amount of text data and information can be easily obtained on the Internet ${ }^{[5]}$. In the field of image processing, more images can be easily processed using cloud infrastructure.

\subsubsection{On-demand service}

End users can choose service requirements according to their own business functions, and they pay according to their own consumption. This service provides end users with virtually unlimited 
computing infrastructure. Therefore, end users can automatically utilize cloud resources without any human interaction.

\section{Cloud computing in universities}

\subsection{Computing Environment Virtualization}

Because each discipline has an independent experimental environment, when the computing environment supports research projects in multiple disciplines, the computing environment that universities sometimes use will suffer from low performance, slow data access, and low throughput. Through cloud computing technology and its virtualization characteristics, organizations can build multiple virtual computing environments that are managed in parallel. The time required to deploy each environment is not long, and Beijing University of Technology completed it in about 30 minutes. The characteristics of virtualization have enabled Beijing University of Technology to save $35 \%$ to $50 \%$ in terms of physical resource cost, performance and throughput ${ }^{[6]}$, and the computing environment has been enhanced.

\subsection{Elastic cloud storage}

Elastic cloud storage enables universities to meet precise storage needs in minutes. No bids, no purchases are required in this process, it is time consuming and requires no additional IT staff to support the infrastructure. It will also be done quickly if the capacity is reduced. It just stopped the vendor's cloud service ${ }^{[7]}$. The use of Amazon S3 for elastic storage by Stanford University and the University of Texas is a strategic development in computing environments. It improves the security and accessibility of computing environments while reducing costs.

\subsection{Cloud-integrated email services}

For large universities with dozens of branches, providing, managing and supporting email services at all remote locations in a timely manner is a significant challenge. At the same time, it is also a challenge to provide efficient backup services to all sites regularly and accurately. Traditionally, maintaining and supporting servers at these remote locations was done one by one by going to these sites ${ }^{[8]}$. This requires a lot of time, effort and cost. By building a unified, centrally located private cloud environment consisting of several servers at the headquarters, the cloud-based email service is a good solution.

From the previous introduction, it is clear that cloud computing technology has improved the performance of the university. In addition, cloud computing plays an important role in reducing costs ${ }^{[8]}$, especially for universities with limited budgets, using cloud computing solutions is critical.

\section{Challenges for universities to adopt cloud computing}

Although cloud computing has many advantages, it also has some disadvantages. Universities face some challenges in adopting cloud computing, the main ones are as follows:

\subsection{Security}

This is the most important factor influencing the use of cloud computing, not only in universities, but in all industries. The management of the university is concerned about the confidentiality and privacy of the data ${ }^{[9]}$. Don't trust vendor statements about security issues. 


\subsection{Bandwidth}

In the case of insufficient Internet bandwidth, since cloud computing is an Internet-based service, especially in the public cloud computing model ${ }^{[10]}$, it will be difficult to provide educational services.

\section{Conclusion}

Cloud computing in universities is still in its infancy compared to other industries, but it will continue to evolve over time. The adoption of cloud computing can help universities to focus on key teaching-related goals with minimal cost. Adoption of cloud computing in universities is necessary. Cloud computing technology plays an important role in reducing costs while improving university performance. Challenges affecting the adoption of cloud computing in universities mainly include security, Internet bandwidth, acceptance by decision makers, and clarity of educational management rules. Security issues remain an important external challenge affecting the adoption of cloud computing in education.

\section{References}

[1] Zheng, L. (2016). Design of cloud computing platform for education laboratory. International Journal of Grid and Distributed Computing, 9(5), 217-228.

[2] C. P. C, O., Justice, N. O., Blessing, C.O. (2021) Application of Cloud Computing Technology in Public Library Services in Nigeria: Strategic Framework for Operational Success. Journal of Academic Librarianship, 47(1), 42-60.

[3] Ali, O., Shrestha, A., Ghasemaghaei, M. et al. . (2021) Assessment of Complexity in Cloud Computing Adoption: a Case Study of Local Governments in Australia. Information Systems Frontiers, 2(1), 162-284.

[4] Mohamed, A., Hamdan, M., Khan, S., Abdelaziz, A., Babiker, S.F.,Imran, M., Marsono, M.N. (2021) Software-Defined Networks for Resource Allocation in Cloud Computing: A Survey. Computer Networks, 195(10.07), 108-151.

[5] Eitedal, M. S., Alhelou, A.M.S., Rashwan, S.S., Abu, N. (2021) The Role of Using Cloud Computing in Improving the Quality of Accounting Education in Palestinian Universities in Light of the Covid-19 Pandemic. Journal of Economics, 3(5), 11-32.

[6] Liu, Y. (2021) The Application of Cloud Computing in College English Teaching. Journal of Physics: Conference Series, 1748(2), 022-002.

[7] Huang, L., Zhou, J., Zhang, G., \& Zhang, M. (2020). Certificateless public verification for data storage and sharing in the cloud. Chinese Journal of Electronics, 29(4), 639-647.

[8] Hamlen, K. W., Kantarcioglu, M., Khan, L., \& Thuraisingham, B. M. (2010). Security issues for cloud computing. International Journal of Information Security and Privacy, 4(2), 36-48.

[9] Tejaswini, K., \& Guruprasad, H. S. (2015). Energy efficiency in cloud computing: a review. International Journal of Scientific Research \& Management Studies, 2(5), 253-259.

[10] Hiran, K.K. (2021) Investigating Factors Influencing the Adoption of IT Cloud Computing Platforms in Higher Education: Case of Sub-Saharan Africa With IT Professional. International Journal of Human Capital and Information Technology Professionals, 7(102), 123-256. 